\title{
NIPPV: Prevalence, Approach and Barriers to Use at Canadian ALS Centres
}

\author{
Benjamin R. Ritsma, Michael J. Berger, David A. Charland, Michael A. Khoury, \\ Joel T. Phillips, Michael J. Quon, Michael J. Strong, Valerie M. Schulz.
}

\begin{abstract}
Objective: The purpose of this study was to evaluate Canadian amyotrophic lateral sclerosis (ALS) centres with respect to: 1) the prevalence of Non-invasive positive pressure ventilation (NIPPV) and invasive mechanical ventilation via tracheostomy (TV) utilization, 2) the approach to NIPPV use, focusing upon the currently employed initiation criteria and 3) the barriers influencing NIPPV administration. Methods: A descriptive survey research design aimed to obtain quantitative data and open-ended responses from an active physician at each of the 15 multidisciplinary Canadian ALS centres. Results: The principal findings of this study were: 1) NIPPV and TV are used in $18.3 \%$ and $1.5 \%$ of patients at Canadian ALS centres, respectively, 2) symptoms of respiratory insufficiency, namely orthopnea (clinical significance rated at 9.00/10 \pm 1.48 ), dyspnea $(8.27 \pm 1.95)$ and morning headache $(7.55 \pm 1.21)$ are the most significant indicators for NIPPV initiation, 3) the primary barriers to NIPPV utilization are patient intolerance (70\% of centres) and inaccessibility of respirologists and ventilation technologists (50\% of centres). Conclusions: Variability in NIPPV use has an impact upon the management of Canadian ALS patients. The establishment of more definitive NIPPV initiation criteria, emphasizing respiratory symptoms, and the attenuation of barriers to NIPPV use should be targeted so as to ensure optimal care for all ALS patients.
\end{abstract}

RÉSUMÉ: La NIPPV : prévalence, approche et freins à son utilisation dans les centres canadiens de traitement de la SLA. Objectif : Le but de cette étude était d'examiner dans les centres canadiens de traitement de la SLA : 1) la prévalence de NIPPV (ventilation non invasive en pression positive) et de l'utilisation de la ventilation mécanique avec trachéotomie (VT); 2) l'approche à l'utilisation de NIPPV ciblant les critères utilisés actuellement pour la commencer et 3) les freins à son utilisation. Méthodes : Nous avons utilisé un plan d'enquête descriptive dont le but était d'obtenir des données quantitatives et des réponses ouvertes des médecins pratiquant dans chacun des 15 centres multidisciplinaires canadiens de traitement de la SLA. Résultats : Les constatations principales de cette étude sont les suivantes : 1) la NIPPV et la VT sont utilisées respectivement chez 18,3\% et $1,5 \%$ des patients des centres canadiens de traitement de la SLA; 2 ) les symptômes d'insuffisance respiratoire, soit l'orthopnée (évaluée au point de vue clinique à $9,00 / 10 \pm 1,48)$, la dyspnée $(8,27 \pm 1,95)$ et la céphalée matinale $(7,55 \pm 1,21)$ sont les indicateurs les plus importants pour le début d'une NIPPV; 3) Les principales entraves à l'utilisation de la NIPPV sont l'intolérance du patient (70\% des centres) et l'absence de pneumologues et de techniciens en assistance respiratoire (50\% des centres). Conclusions : La variabilité de l'utilisation de la NIPPV a un impact sur le traitement des patients canadiens atteints de SLA. Afin d'assurer la prise en charge optimale de tous les patients atteints de SLA, il faudra procéder à l'élaboration de critères de référence plus précis pour débuter la NIPPV en mettant l'emphase sur les symptômes respiratoires et tenter d'atténuer les barrières à son utilisation .

Can. J. Neurol. Sci. 2010; 37: 54-60

Non-invasive positive pressure ventilation (NIPPV) has become the accepted standard of care for the initial management of respiratory insufficiency in amyotrophic lateral sclerosis $(\mathrm{ALS})^{1,2}$. A series of prospective studies have shown that when tolerated for greater than four hours per day, NIPPV therapy prolongs survival, increases quality of life and reduces decline in pulmonary function ${ }^{3-7}$. In addition, a recent randomized controlled trial has supported NIPPV's survival and quality of life benefit in a subset of ALS patients with good bulbar function ${ }^{8}$. Nevertheless, considerable variability in the prevalence of NIPPV use has been documented in the international ALS patient population. A survey of 265 British neurologists reported that approximately $2.6-3.5 \%$ of patients were utilizing NIPPV, with $30 \%$ of all referrals coming from three physicians ${ }^{9}$. In addition, a questionnaire completed by 110
European ALS specialists revealed that only $56 \%$ of centres routinely offered NIPPV ${ }^{10}$. Recent U.S. data estimate that 7 $15.6 \%$ of ALS patients utilize NIPPV ${ }^{11,12}$, and further analyses of the ALS CARE Database have suggested that NIPPV use is

From the Schulich School of Medicine \& Dentistry (BRR, MJB, DAC, MAK, JTP, MJQ, MJS, VMS), School of Kinesiology, Faculty of Health Sciences (MJB), Department of Clinical Neurological Sciences (MJS), Department of Anesthesia \& Perioperative Medicine (VMS), The University of Western Ontario, London, Ontario, Canada.

Received May 22, 2009. Final Revisions Submitted July 27, 2009. Correspondence to: Michael J. Strong, Room C7-120, UH-LHSC, 339 Windermere Road, London, Ontario, N6A 5A5, Canada. 
sub-optimal ${ }^{13-15}$. In Japan, invasive mechanical ventilation via tracheostomy (TV) is commonly used in place of NIPPV ${ }^{16}$. Currently, the prevalence of NIPPV prescription at Canadian ALS centres is unknown.

A primary source of variability in the rate of NIPPV institution arises from the lack of consensus regarding the optimal criteria for its initiation ${ }^{17,18}$. The American Academy of Neurology Practice Parameter for ALS suggests that NIPPV be initiated when forced vital capacity (FVC) falls below $50 \%$ of its predicted value and/or upon the development of respiratory symptoms ${ }^{2}$. However, in spite of this practice parameter, only $36 \%$ of patients in the ALS CARE Database with FVC $<50 \%$ predicted were receiving NIPPV treatment ${ }^{14}$. In addition, an FVC $<50 \%$ predicted may not be the most sensitive indicator of the onset of respiratory insufficiency. Indeed, several observational studies have reported that an earlier initiation of NIPPV, such as upon first evidence of nocturnal desaturation, provides a greater survival benefit ${ }^{19,20}$.

Another potential explanation for the variation in NIPPV prescription stems from patient and health care system related issues that may prevent adherence to clinical practice guidelines ${ }^{1,2,4,10,15,21-23}$. Important patient-induced barriers include the decision to decline NIPPV use and NIPPV intolerance due to factors such as interface discomfort, claustrophobia or anxiety, disease progression, bulbar muscle involvement, difficulty in secretion management and frontotemporal dysfunction. Both human resource and financial impediments have also been identified in the context of health care professional (e.g. respiratory therapist), caregiver and equipment availability ${ }^{10,21}$.

Current U.S. ${ }^{2}$ and European ${ }^{1}$ guidelines recommend that ALS patients be offered an informed choice with respect to TV and palliative care when NIPPV therapy can no longer be tolerated (e.g. loss of bulbar tone or difficulties with secretion management) or becomes ineffective due to disease progression. TV second-line use is attributed to its high cost and significant emotional, social, and financial impact on patients and their caregivers $^{1,2}$. Although TV can prolong survival and may be deemed acceptable by some patients and caregivers, its use has often been associated with ethical dilemmas, including to ventilation withdrawal decisions and locked-in syndrome. As a result, the majority of patients refuse its use ${ }^{24}$ and current U.S. $(2.1 \%-3 \%)$ and European $(2.6 \%)$ prescription rates are accordingly low. However, in Japan, invasive ventilators (TV or ventilation via intubation) are more commonly used than NIPPV ${ }^{16}$. Canadian TV figures have yet to be determined.

Given these issues, the treatment of respiratory compromise in ALS patients continues to be empiric and variable in nature ${ }^{24}$. As this variability has an impact upon patient care, it is important to assess individual physician's management strategies. Because

Table 1: Questionnaire content

\begin{tabular}{|c|c|c|}
\hline Sub-category & Quantitative Content & $\begin{array}{c}\text { Open-Ended } \\
\text { Content/Questions }\end{array}$ \\
\hline $\begin{array}{l}\text { 1. Physician } \\
\text { characteristics }\end{array}$ & - medical specialty of respondent & $\cdot \mathrm{N} / \mathrm{A}$ \\
\hline 2. Demographic data & $\begin{array}{l}\text { - number of ALS patients } \\
\text { - percentage of ALS patients using NIPPV } \\
\text { - average duration of NIPPV use/patient } \\
\text { - method of NIPPV most commonly prescribed } \\
\text { - institutional protocol for NIPPV prescription (yes/no) } \\
\text { - percentage of ALS patients using TV }\end{array}$ & $\cdot \mathrm{N} / \mathrm{A}$ \\
\hline 3. Access to care & $\begin{array}{l}\text { - proportion of ALS patients unable to receive NIPPV } \\
\text { despite an indication for its use }\end{array}$ & - barriers to NIPPV use \\
\hline $\begin{array}{l}\text { 4. Physician perception } \\
\text { of NIPPV usage } \\
\text { 5. Physician Approach } \\
\text { to the Initiation of } \\
\text { NIPPV }\end{array}$ & $\begin{array}{l}\text { - perception of prevalence of NIPPV use compared to } \\
\text { other centres (above average, average, or below average) }\end{array}$ & - explanation of reasoning \\
\hline a. Investigations & $\begin{array}{l}\text { - investigations (and thresholds) used in the decision to } \\
\text { initiate NIPPV: FVC, supine FVC, SNP, nocturnal } \\
\text { oximetry, morning blood gas (e.g. } \mathrm{PCO}_{2} \text { ), MIP, Other }\end{array}$ & $\begin{array}{l}\text { - strongest indication for } \\
\text { initiation of NIPPV }\end{array}$ \\
\hline b. Clinical Presentation & $\begin{array}{l}\text { - degree of importance ascribed to symptoms used in } \\
\text { decision to initiate NIPPV: dyspnea, orthopnea, disturbed } \\
\text { sleep not due to pain, morning headache, poor } \\
\text { concentration, loss of appetite, excessive daytime } \\
\text { sleepiness, other }\end{array}$ & $\cdot \mathrm{N} / \mathrm{A}$ \\
\hline 6. General Comments & $\cdot \mathrm{N} / \mathrm{A}$ & - provide comments \\
\hline
\end{tabular}

A summary of questionnaire content is providedNIPPV: non-invasive positive pressure ventilation; TV: invasive mechanical ventilation via tracheostomy; FVC: forced vital capacity; SNP: sniff nasal pressure; MIP: maximal inspiratory pressure; N/A: not assessed. 
the provision of health care to Canadian ALS patients is largely within the purview of academic tertiary care referral centres, in which multidisciplinary teams exist, we have examined the prevalence of NIPPV and TV therapy at Canadian ALS centres, the currently employed NIPPV initiation criteria at these centres and assessed the barriers influencing Canadian NIPPV administration.

\section{Material AND MethodS}

A descriptive survey research design was used to assess the prescription practices of Canadian ALS physicians. Questions were formulated using the following phases of survey development: 1) specification of measurement goals, 2) item generation, 3) item reduction and 4) questionnaire formatting. A physician expert in the field of ALS care assessed the content of the questionnaire for relevance and clarity prior to its distribution. The questionnaire consisted of both quantitative data collection and qualitative open-ended questions. Its content was grouped into six subcategories (Table 1). In subcategories four and five, the list of investigations and symptoms reflected their ascribed importance in current clinical practice guidelines $^{1,2}$.

The study utilized purposive sampling of an active physician at each of the 15 multidisciplinary Canadian ALS centres. The questionnaire was initially distributed to the Medical Director or a representative staff physician of centres attending the ALS Society of Canada 2008 Research Forum. After two months, the questionnaire was mailed to centres from which a response to the initial distribution had not been received. This second iteration targeted all 15 multidisciplinary Canadian ALS centres, as identified by Shoesmith and Strong ${ }^{23}$ and the ALS Society of Canada website at the time of survey development. Therefore, this distribution phase facilitated data collection from centres not attending the Research Forum.

Investigators were blinded as to the identification of the responding centre. Quantitative data was analyzed with descriptive statistics where appropriate, while qualitative open- ended questions were analyzed by the grouping of responses into emerging themes. This study received ethics approval from The University of Western Ontario, Research Ethics Board and informed consent was assumed upon completion of the questionnaire.

\section{RESULTS}

An active physician at 11 of the targeted 15 multidisciplinary Canadian ALS centres completed and submitted the questionnaire (response rate $=73.3 \%$ ). The results are presented in the order of their appearance in the questionnaire (Table 1). Eight respondents were neurologists, two were physiatrists and one was a respirologist. Demographic data for the 11 responding Canadian ALS centres is summarized in Table 2.

In terms of access to care, six centres reported the ability to provide NIPPV to all patients who meet their particular initiation criteria and desire its use. However, three of these centres noted delays (e.g. up to six months at one location) in acquiring NIPPV access. Three centres indicated that NIPPV provision is not possible in up to $10-30 \%$ of patients who satisfy their initiation criteria, while in those for whom NIPPV can be provided there can be as much as a "six to eight week wait" to obtain the appropriate equipment. One centre noted that the pneumonologist would be more proficient in answering this question and one centre did not provide a response. Barriers to NIPPV prescription and utilization are highlighted in Table 3.

Four centres cited their perception of NIPPV usage as 'above average' according to the following rationale: 1) "good coordination between neurology and respirology", 2) "three different routes available for obtaining machines so most get it" and 3) "don't adhere to strict objective guidelines so it will be offered to patients with test changes even if asymptomatic, or if symptomatic with no test changes." Five respondents perceived their rate of prescription to be 'average'; with one stating that "recent changes with [our] respirologist may have decreased the frequency of my referrals somewhat, to those most clearly in need" while another was "uncertain [as to] what occurs at other

Table 2: Demographic data

$\begin{array}{ll}\text { Total number of ALS patients } & 939 \\ \text { Mean number of ALS patients / centre } & 85.4 \pm 67.4 \\ \text { Total percentage of ALS patients using NIPPV } & 18.3 \% \\ \text { Mean percentage of ALS patients using NIPPV / centre } & 23.4 \pm 11.3 \% \\ \text { Mean duration of NIPPV use / patient } & 8.8 \pm 5.2 \text { months } \\ & \text { (minimum=2; } \\ & \text { maximum }=18) \\ \text { Number of centres using BIPAP as primary method of NIPPV } & 10 / 10 \text { respondents } \\ \text { Number of centres adhering to specific institutional protocol with regards to NIPPV } & 0 / 11 \text { respondents } \\ \text { prescription } & 1.5 \% \\ \text { Total Percentage of ALS patients using TV } & 0.5(0-3.3) \\ \text { Median of ALS patients using TV / centre } & (5 \text { centres }=0 \text { patients) } \\ \end{array}$

A summary of demographic data is provided. Means ( \pm standard deviations) and medians (interquartile range) are reported where appropriate. NIPPV: non-invasive positive pressure ventilation; BIPAP: bi-level intermittent positive airway pressure; TV: invasive mechanical ventilation via tracheostomy. 
centres". A single centre perceived their prescription frequency to be 'below average' due to NIPPV intolerance as "most patients don't like it."

\section{Physician approach to the initiation of NIPPV}

Investigations: A summary of the investigations used in the decision to initiate NIPPV is presented in Figure 1. Forced vital capacity thresholds providing a basis for the initiation of NIPPV treatment differed between centres, with 2 centres using an FVC at $<50 \%, 4$ at $<60 \%$ and 1 at $<70 \%$ of predicted. Nocturnal oximetry thresholds employed for NIPPV initiation were provided by 5 of 11 responding centres with 3 centres citing thresholds of $<90 \%$ and 2 citing a threshold of $<89 \%$ oxygen saturation. Data illustrating the investigation that is the strongest indication for the decision to introduce NIPPV is provided in Figure 2.

Clinical presentation: The clinical importance ascribed to symptoms of respiratory compromise in NIPPV initiation decisions was rated on a 10 - point scale and is presented in Figure 3. Other symptoms, each indicated by a single centre in an empty space provided on the questionnaire, included: increased malaise without explanation (10/10), morning confusion $(8 / 10)$, increased cough $(7 / 10)$, reduced sentence length (5/10) and increased nightmares (3/10).

\section{Discussion}

This research is of importance as Canadian NIPPV practices in ALS treatment have been predominantly unaddressed and such an assessment may bring forth a meaningful contribution to the international discussion regarding NIPPV therapy for ALS

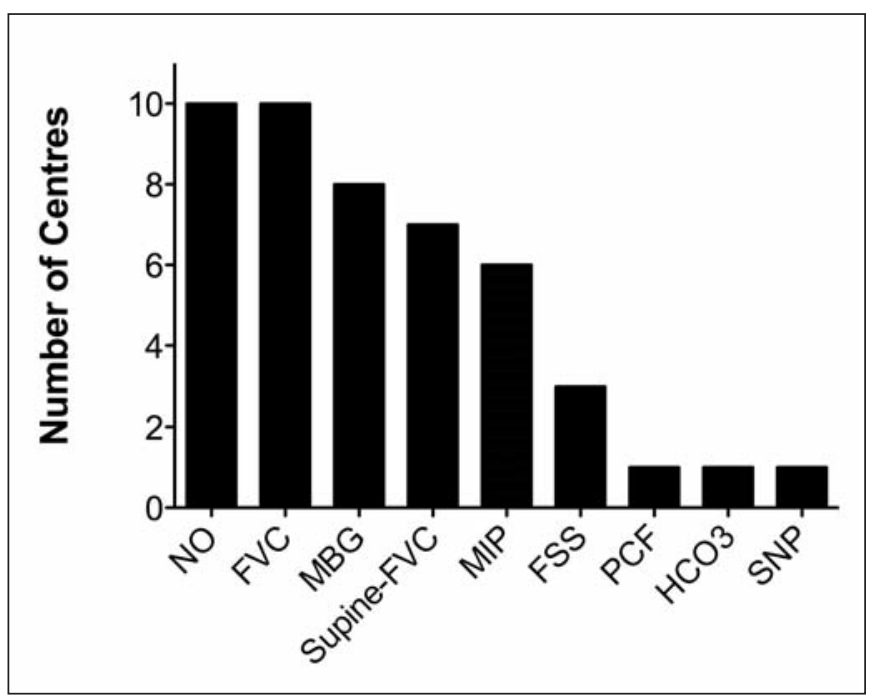

Figure 1: Investigations used in the decision to initiate NIPPV. The number of responding centres ( $y$-axis) utilizing a particular investigation (x-axis) is presented. NO: nocturnal oximetry; FVC: forced vital capacity; MBG: morning blood gases; MIP: maximal inspiratory pressure; FSS: formal sleep study; PCF: peak cough flows; $\mathrm{HCO}_{3}$ : blood bicarbonate; SNP: sniff nasal pressure.

\section{Table 3: Barriers to NIPPV ulilization}

\section{Number of}

Barriers

Canadian

ALS centres

\begin{tabular}{ll}
\hline $\begin{array}{l}\text { Patient intolerance (including bulbar } \\
\text { involvement/symptoms) }\end{array}$ & 7 \\
Lack of access to a respirologist \\
$\begin{array}{l}\text { and/or ventilation technologist } \\
\text { Financial (i.e. cost of ventilatory } \\
\text { equipment) }\end{array}$ & 5 \\
$\begin{array}{l}\text { Patient declines NIPPV treatment } \\
\text { Lack of available NIPPV machines }\end{array}$ & 3 \\
$\begin{array}{l}\text { Lack of caregiver help } \\
\text { Lack of support in rural communities }\end{array}$ & 1 \\
Failure of patient to meet provincial & 1 \\
criteria for NIPPV machine allocation & 1
\end{tabular}

Specific barriers are listed in the left-hand column and the frequency at which they were reported is listed in the right-hand column (10/11 survey respondents completed the question).

patients. This study includes data from 11 of the 15 multidisciplinary Canadian ALS centres (939 Canadian ALS patients) with a response rate of $73.3 \%$. The ensuing discussion will focus upon; 1) NIPPV and TV prevalence, 2) the approach to NIPPV use and 3) the barriers to NIPPV administration at these centres.

According to current clinical practice guidelines, the management of respiratory insufficiency in ALS patients should include the administration of NIPPV as a primary treatment modality ${ }^{1,2}$. Currently, $18.3 \%$ of patients at the responding Canadian ALS centres are receiving NIPPV therapy. Although variability exists, Canadian centres have a higher prevalence of NIPPV prescription with respect to their U.S. $(7-15.6 \%)^{11,12}$, U.K. $(2.6-3.5 \%)^{9}$ and European ${ }^{10}$ counterparts.

Subsequent to NIPPV therapy, ALS patients are to be offered an informed choice with regards to TV and palliative care treatment options ${ }^{1,2}$. This study found that $1.5 \%$ (12 patients in total) of Canadian ALS centre patients were receiving TV, with five centres having no patients receiving this form of treatment. These figures are slightly lower, yet similar, to previously collected U.S. $(2.1-3 \%)^{11,13,15}$ and European $(2.6 \%)^{10}$ data. Conversely, Japanese ALS patients are more likely to receive TV than NIPPV in response to respiratory insufficiency ${ }^{16}$. Thus, our TV numbers appear to support a general trend towards a more restricted use of invasive mechanical ventilation.

At Canadian centres, patient symptoms are of primary importance in the decision to introduce NIPPV, with the presence 


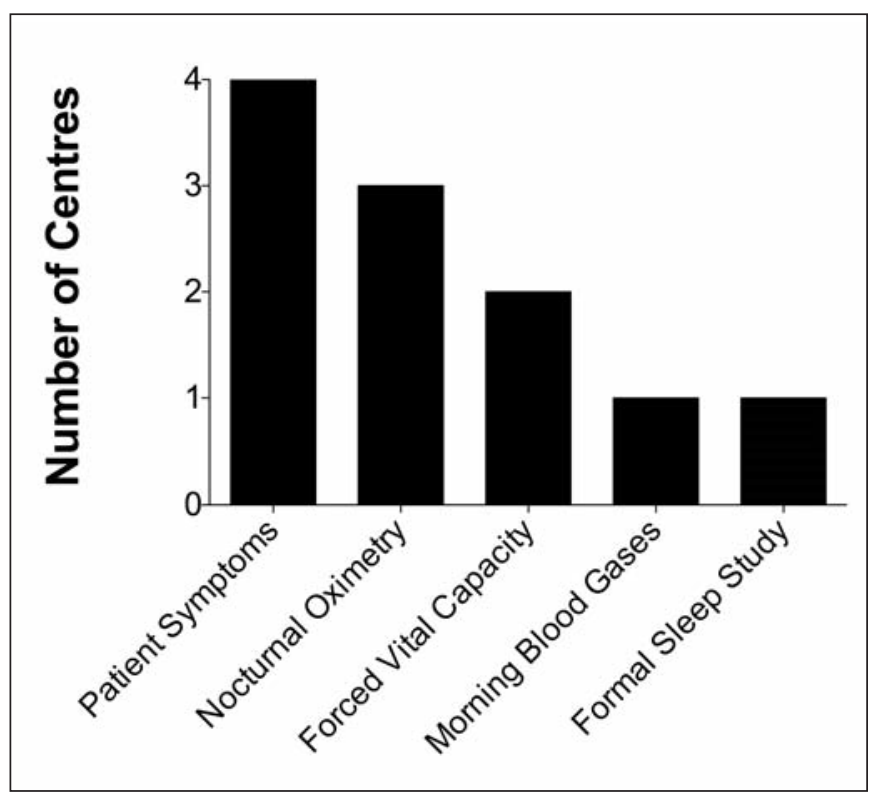

Figure 2: Most important investigation. Respondents were asked to cite the investigation ( $x$-axis) that provides the strongest indication to initiate NIPPV.

of orthopnea, dyspnea and morning headache amongst the strongest indicators for its initiation. This is consistent with current practice trends as recent findings have indicated that orthopnea and dyspnea are most heavily weighted by U.K. neurologists when considering NIPPV initiation ${ }^{9}$, and that the presence of orthopnea is the best predictor of benefit from, and compliance with, NIPPV ${ }^{14,25}$. Moreover, due to the strong correlation between orthopnea, dyspnea and NIPPV use ${ }^{14,18}$, it has been suggested that pulmonary function tests be considered secondary to symptoms of respiratory dysfunction ${ }^{18}$. The responses to the survey's open-ended questions provided further support for the primary importance of symptom assessment. For example, one respondent replied that NIPPV is offered if a patient is "...symptomatic despite the absence of hard objective test changes". Another stated "...I place much more reliance on the patient's symptoms than I do on pulmonary function tests...". Moreover, symptoms were cited as "...most important in the decision to start BiPAP [bi-level intermittent positive airway pressure]."

The significance of these comments is strongly corroborated by an unexpected trend in the responses to open-ended questions regarding investigations of respiratory function (Table 1, section 5a). When asked to identify the investigation that serves as the strongest indication in their decision to initiate NIPPV, patient symptoms were reported to be the most critical (Figure 2). Similarly, when asked to report the combination of tests most frequently used in the institution of NIPPV, patient symptoms were cited with the second greatest frequency. In each instance, the question was explicit in its instructions to identify an investigation or a series of tests, as symptoms were assessed in a separate survey section. Thus, the fact that patient symptoms

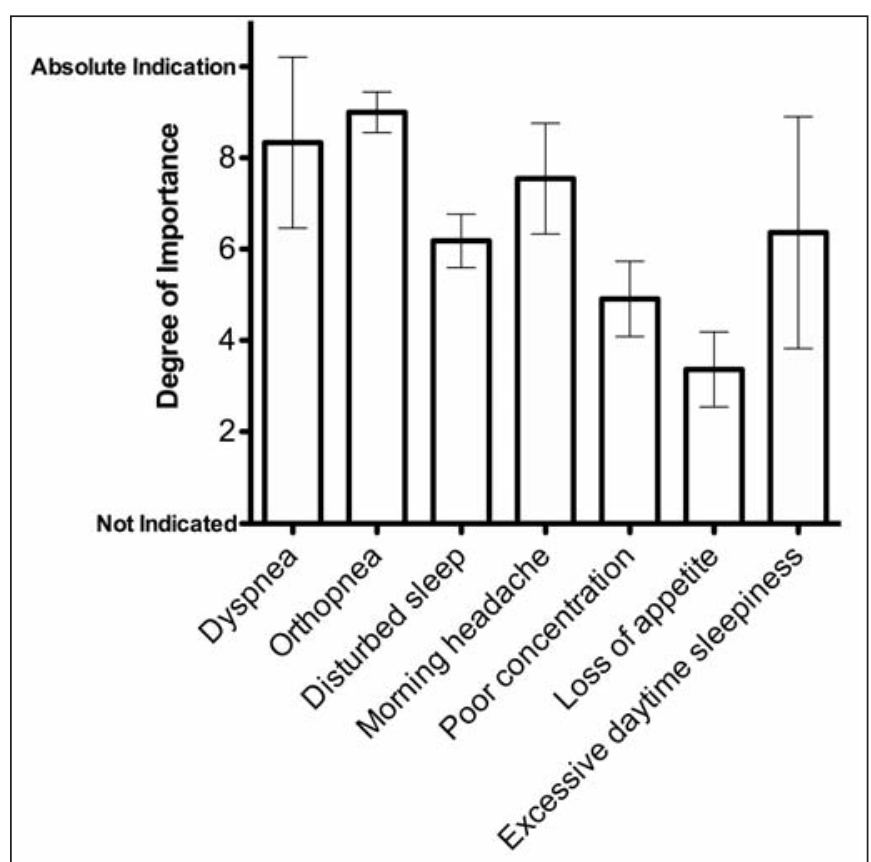

Figure 3: Degree of importance ascribed to clinical symptoms. The mean degree of importance ( $y$-axis) ascribed to clinical symptoms ( $x$ axis) (1=not important, 10=absolute importance) is presented.

were so strongly represented provides further evidence as to their central importance in NIPPV initiation.

With respect to investigations used in the decision to implement NIPPV, nocturnal oximetry, FVC and morning blood gases were used at the greatest number of centres. Other tests employed by multiple respondents include supine FVC, maximal inspiratory pressure and formal sleep studies, whereas sniff nasal pressure, peak cough flow rate and blood bicarbonate levels were each used in a single centre. This variability suggests a lack of consensus as to the most useful investigations and is likely a source of the discrepancy in NIPPV prescription rates between centres. It is interesting to note the rather widespread use of supine FVC ( 7 of 11 centres). As supine FVC $<75 \%$ predicted has been shown to be more sensitive and specific in detecting early diaphragmatic weakness, some authors have proposed its superiority to upright $\mathrm{FVC}^{26}$.

In centres frequently using FVC to guide initiation of NIPPV, variability was reported with respect to the thresholds used, as values range from $<70 \%$ to $<50 \%$ of predicted. Although the current guidelines indicate the use of FVC $<50 \%$ in the U.S $S^{1,2}$ and FVC $<80 \%$ in Europe ${ }^{1,2}$, the most optimal threshold remains unknown and its subsequent application continues to be empiric $^{11}$. Given this lack of definitive criteria abroad and the absence of Canadian practice guidelines, Canadian ALS physicians have also adapted an empiric approach, basing NIPPV initiation decisions upon each patient's clinical context, their own clinical experience and the influence of systemic pressures. Among those investigations listed as the strongest 
indication for the prescription of NIPPV, nocturnal oximetry ranked highest, ahead of FVC. This finding is in keeping with studies suggesting that FVC may not be the most sensitive indicator for NIPPV initiation ${ }^{18-20}$. Currently utilized Canadian nocturnal oximetry threshold values are more consistent and they reflect those figures referred to in previous investigations (e.g. $<80-90 \%$ oxygen saturation $)^{20,27,28}$. Therefore, secondary to symptoms, Canadian centres appear to be placing greater importance on investigations (i.e. nocturnal oximetry) and test thresholds (i.e. before $\mathrm{FVC}<50 \%$ ) that facilitate an earlier implementation of NIPPV.

According to respondents, no specific institutional protocol is in place for the administration of NIPPV in ALS patients. Similarly, no organized, structured pulmonary protocol exists in most U.S. centres ${ }^{11}$. Systematic application of a standardized protocol has been shown to reduce utilization of TV, decrease the incidence of NIPPV initiation in an acute setting and increase survival in non-bulbar patients ${ }^{27}$. Farrero et al suggest that these benefits were attributable to an earlier application of NIPPV ${ }^{27}$. Although it is difficult to assume that this single study would generalize to the Canadian setting, future investigation could examine the role and potential value of establishing an institutional protocol at Canadian ALS centres.

Subsequent to the decision to introduce NIPPV, substantial variability in the average duration of NIPPV use was noted between Canadian ALS centres (mean of $8.8 \pm 5.2$ months, with a range from 2-18 months). This finding is consistent with the empiric nature of NIPPV initiation and withdrawal ${ }^{24}$. Although dissimilarities in barriers to NIPPV use are likely to assume partial responsibility, further study is required to more fully elucidate the reasons for and the impact of this variability. Moreover, an analysis of the currently employed approach to NIPPV withdrawal at Canadian ALS centres could prove quite meaningful.

The most commonly encountered impediments at Canadian ALS centres to the institution of NIPPV are patient intolerance and poor coordination of care due to a lack of access of health care professionals, most notably respirologists and/or ventilation technologists. Additionally, issues related to financing ventilatory equipment, patient decision to decline NIPPV treatment, the availability of BiPAP machines, access to caregiver help, support in rural communities and patient ability to meet provincial criteria for NIPPV machine allocation were reported.

As respondents emphasized "tolerance of the interface" as the primary barrier to NIPPV administration and previous research groups have described similar findings $\mathrm{s}^{1,2,23}$, this study provides further support for the importance of future investigation targeting this issue. Although tolerance of the treatment modality is a universal and important issue, it certainly is not specific to Canadian ALS centre care. Of interest to this study, is the identification of barriers subject to regional systemic pressures within the context of the Canadian health care system. Most notably, the second most frequent impediment to NIPPV utilization at Canadian centres is coordination of care. This hindrance focuses upon the lack of timely access to respirologists and/or ventilation technologists. For example, one respondent replied that their primary barrier is a "...delay to be seen by a pneumonologist... and a lack of ventilation technologists to follow-up at home..." In addition to coordination of care constraints, the issue of accessibility extends to finances and equipment. At three centres, "cost is an issue..." in obtaining NIPPV machines and even when funds are in place, one respondent indicated that a "lack of readily available BiPAP units...for immediate use by patients" results in "...a six to eight week wait to get the unit from the [provincial] ventilator pool...". At another centre, this delay for access to NIPPV from the "provincial home ventilator program...is up to six months". Collectively, these barriers were cited as cause for delayed initiation of NIPPV. As research has advocated for earlier initiation times, such a postponement may result in less than optimal care for some ALS patients ${ }^{19,20,27}$.

As evidenced by inter-center variability in NIPPV prescription rates $(23.4 \pm 11.3 \%)$ and open-ended responses, the impediments to NIPPV use are by no means equally apparent at all centres. For instance, one physician perceived effective use of NIPPV at their centre due to "good coordination between respirologist and neurologist..." in the context of "...same day clinics." In another centre the respondent had "...access to NIPPV through three routes..." which ensured that "...patients do get the machines." While an extensive examination of the reasons for this potential discrepancy is beyond the scope of this paper, it seems evident that systemic and institutional factors have an important contribution. For instance, within the Canadian health care system, each province and, to a certain extent, each institution sets its own priorities with regards to health care delivery ${ }^{29}$. Moreover, provincial ALS Societies influence important aspects of patient care provision and operate independently in determining their contribution to the province's ALS centres and patients. As such, Canadian ALS centres may receive differing levels of support in terms of the human resources, finances, and equipment necessary for NIPPV administration.

One potential limitation of this study is that data from four of Canada's 15 multidisciplinary ALS centres has not been acquired. Although the response rate appears adequate, this missing data from a small sample size may hinder the validity for broad generalizations. As described above, it is possible that the care provided by non-responding centres differs due to regional systemic factors, including geography, language and heath care resources. A thorough examination of such regional influences was neither feasible given the study's methodology (i.e. survey content and blinded analysis) nor a primary study objective. Nonetheless, open-ended responses did attest to the impact of regional variation and as such further investigation may prove quite useful in optimizing the use of NIPPV in Canada.

In conclusion, this descriptive survey research was able to demonstrate that NIPPV and TV are used in a mean of $18.3 \%$ and $1.5 \%$ of patients at responding Canadian ALS centres, respectively. Physicians at these multidisciplinary centres emphasize patient respiratory symptoms, most notably orthopnea, dyspnea and morning headache, in their decision to initiate NIPPV. This introduction may also be supported by the results of investigations such as nocturnal oximetry, FVC and morning blood gases. Finally, the primary barriers to the provision of NIPPV therapy in Canada are patient intolerance and less than optimal coordination of care due to a lack of access to health care professionals, most commonly respirologists 
and/or ventilation technologists. Similar to the U.S., U.K. and European analysis, there is variability in NIPPV prevalence, approach and barriers to use amongst Canadian ALS centres. A lack of consensus regarding initiation criteria, differing impediments to NIPPV use and their interaction with regional systemic factors appear to contribute to the discrepancy. As this variability has an impact upon the management of Canadian ALS patients, future study should focus upon: 1) establishing more definitive NIPPV initiation criteria, with an emphasis upon symptoms of respiratory insufficiency; and 2) attenuating barriers to NIPPV use, to ensure optimal care for all ALS patients.

\section{ACKNOWLEDGEMENTS}

The authors thank Dr. Timothy J. Doherty for contributions towards study conception and design and Dr. Christen Shoesmith for reviewing survey content. The authors would also like to express sincere thanks to all responding Canadian ALS centres and their patients who have made this study possible.

\section{REFERENCES}

1. Andersen PM, Borasio GD, Dengler R, Hardiman O, Kollewe K, Leigh PN, et al. Good practice in the management of amyotrophic lateral sclerosis: clinical guidelines. An evidencebased review with good practice points. EALSC Working Group. Amyotroph Lateral Scler. 2007; 8(4):195-213.

2. Miller RG, Rosenberg JA, Gelinas DF, Mitsumoto H, Newman D, Sufit R, et al. Practice parameter: the care of the patient with amyotrophic lateral sclerosis (an evidence-based review): report of the Quality Standards Subcommittee of the American Academy of Neurology: ALS Practice Parameters Task Force. Neurology. 1999; 52(7):1311-23.

3. Aboussouan LS, Khan SU, Banerjee M, Arroliga AC, Mitsumoto H. Objective measures of the efficacy of noninvasive positivepressure ventilation in amyotrophic lateral sclerosis. Muscle Nerve. 2001; 24(3):403-9.

4. Aboussouan LS, Khan SU, Meeker DP, Stelmach K, Mitsumoto H. Effect of noninvasive positive-pressure ventilation on survival in amyotrophic lateral sclerosis. Ann Intern Med. 1997; 127(6): 450-3.

5. Kleopa KA, Sherman M, Neal B, Romano GJ, Heiman-Patterson T. Bipap improves survival and rate of pulmonary function decline in patients with ALS. J Neurol Sci. 1999; 164(1):82-8.

6. Lo Coco D, Marchese S, Pesco MC, La Bella V, Piccoli F, Lo Coco A. Noninvasive positive-pressure ventilation in ALS: predictors of tolerance and survival. Neurology. 2006; 67(5):761-5.

7. Pinto AC, Evangelista T, Carvalho M, Alves MA, Sales Luis ML. Respiratory assistance with a non-invasive ventilator (Bipap) in MND/ALS patients: survival rates in a controlled trial. J Neurol Sci. 1995; 129 Suppl:19-26.

8. Bourke SC, Tomlinson M, Williams TL, Bullock RE, Shaw PJ, Gibson GJ. Effects of non-invasive ventilation on survival and quality of life in patients with amyotrophic lateral sclerosis: a randomised controlled trial. Lancet Neurol. 2006; 5(2):140-7.

9. Bourke SC, Williams TL, Bullock RE, Gibson GJ, Shaw PJ. Noninvasive ventilation in motor neuron disease: current UK practice. Amyotroph Lateral Scler Other Motor Neuron Disord. 2002; 3(3):145-9.

10. Borasio GD, Shaw PJ, Hardiman O, Ludolph AC, Sales Luis ML, Silani V. Standards of palliative care for patients with amyotrophic lateral sclerosis: results of a European survey. Amyotroph Lateral Scler Other Motor Neuron Disord. 2001; 2 (3):159-64.
11. Melo J, Homma A, Iturriaga E, Frierson L, Amato A, Anzueto A, et al. Pulmonary evaluation and prevalence of non-invasive ventilation in patients with amyotrophic lateral sclerosis: a multicenter survey and proposal of a pulmonary protocol. J Neurol Sci. 1999; 169(1-2):114-7.

12. Cedarbaum JM, Stambler N. Disease status and use of ventilatory support by ALS patients. BDNF Study Group. Amyotroph Lateral Scler Other Motor Neuron Disord. 2001; 2(1):19-22.

13. Bradley WG, Anderson F, Gowda N, Miller RG. Changes in the management of ALS since the publication of the AAN ALS practice parameter 1999. Amyotroph Lateral Scler Other Motor Neuron Disord. 2004; 5(4):240-4.

14. Jackson CE, Lovitt S, Gowda N, Anderson F, Miller RG. Factors correlated with NPPV use in ALS. Amyotroph Lateral Scler. 2006; 7(2):80-5.

15. Lechtzin N, Wiener CM, Clawson L, Davidson MC, Anderson F, Gowda N, et al. Use of noninvasive ventilation in patients with amyotrophic lateral sclerosis. Amyotroph Lateral Scler Other Motor Neuron Disord. 2004; 5(1):9-15.

16. Borasio GD, Gelinas DF, Yanagisawa N. Mechanical ventilation in amyotrophic lateral sclerosis: a cross-cultural perspective. J Neurol. 1998; 245 Suppl 2:S7-12; discussion S29.

17. Bourke SC, Gibson GJ. Non-invasive ventilation in ALS: current practice and future role. Amyotroph Lateral Scler Other Motor Neuron Disord. 2004; 5(2):67-71.

18. Sivak ED, Shefner JM, Mitsumoto H, Taft JM. The use of noninvasive positive pressure ventilation (NIPPV) in ALS patients. A need for improved determination of intervention timing. Amyotroph Lateral Scler Other Motor Neuron Disord. 2001; 2 (3): $139-45$.

19. Lechtzin N, Scott Y, Busse AM, Clawson LL, Kimball R, Wiener $\mathrm{CM}$. Early use of non-invasive ventilation prolongs survival in subjects with ALS. Amyotroph Lateral Scler. 2007; 8(3):185-8.

20. Pinto A, de Carvalho M, Evangelista T, Lopes A, Sales-Luis L. Nocturnal pulse oximetry: a new approach to establish the appropriate time for non-invasive ventilation in ALS patients. Amyotroph Lateral Scler Other Motor Neuron Disord. 2003; 4 (1):31-5.

21. Heiman-Patterson TD, Miller RG. NIPPV: a treatment for ALS whose time has come. Neurology. 2006; 67(5):736-7.

22. Hillberg RE, Johnson DC. Noninvasive ventilation. N Engl J Med. 1997; 337(24):1746-52.

23. Shoesmith CL, Strong MJ. Amyotrophic lateral sclerosis: update for family physicians. Can Fam Physician. 2006; 52(12):1563-9.

24. Mitchell JD, Borasio GD. Amyotrophic lateral sclerosis. Lancet. 2007; 369(9578):2031-41.

25. Bourke SC, Bullock RE, Williams TL, Shaw PJ, Gibson GJ. Noninvasive ventilation in ALS: indications and effect on quality of life. Neurology. 2003; 61(2):171-7.

26. Lechtzin N, Wiener CM, Shade DM, Clawson L, Diette GB. Spirometry in the supine position improves the detection of diaphragmatic weakness in patients with amyotrophic lateral sclerosis. Chest. 2002; 121(2):436-42.

27. Farrero E, Prats E, Povedano M, Martinez-Matos JA, Manresa F, Escarrabill J. Survival in amyotrophic lateral sclerosis with home mechanical ventilation: the impact of systematic respiratory assessment and bulbar involvement. Chest. 2005; 127 (6):2132-8.

28. Gelinas D. Respiratory complications of amyotrophic lateral sclerosis: symptoms, signs, diagnosis and treatment. Continuum: lifelong learning in neurology. 2002; 8(4):110-24.

29. Health Canada. Canadian Health Act - Annual Report 2006-07. Ottawa: Heath Canada; 2007. p. 1. 